\title{
FINITE GROUPS AS AUTOMORPHISM GROUPS OF ORTHOCOMPLEMENTED PROJECTIVE PLANES
}

\author{
RICHARD J. GREECHIE \\ (Received 18 December 1975; revised 8 March 1977) \\ Communicated by J. N. Crossley
}

\begin{abstract}
A construction is given for a non-desarguesian projective plane $P$ and an absolute-point free polarity on $P$ such that the group of collineations of $P$ which commute with the polarity is isomorphic to an arbitrary preassigned finite group.
\end{abstract}

Sabidussi (1957), in extending the fundamental work of Frucht (1949), proved that every finite group $G$ (of order at least 2) may be represented as the automorphism group of a regular graph of degree 5 . Schrag $(1971,1976)$ showed how to parlay this result into a representation of $G$ as the automorphism group of some orthomodular lattice $L$. In this paper we show that $L$ may be chosen to be an irreducible orthocomplemented modular lattice of height 3 , herein called an orthocomplemented projective plane. In fact we shall prove the following result.

THEOREM. Let $G$ be a finite group. There exists a non-desarguesian projective plane $P$ and an absolute-point free polarity 'on $P$ and an injection $\varphi: G \rightarrow \operatorname{coll}(P)$ of $G$ into the collineation group of $P$ such that image $(\varphi)=\left\{\alpha \in \operatorname{coll}(P) \mid \alpha^{\prime}={ }^{\prime} \alpha\right\}$.

The idea of the proof is to look at Schrag's orthomodular lattice $L$ with $\operatorname{Aut}_{\perp}(L) \cong G$ and observe that $\operatorname{Aut}_{\perp}(L)=\operatorname{Aut}(L)$, that is, every lattice automorphism of $L$ preserves the orthocomplementation. We then construct a wide cubic structure space (see Greechie (1974)) from the elements of $L$ of height 1 or 2. We extend this to a larger such structure which is also a confined configuration (see Greechie (1974)) by adjoining a wide cubic structure space with no automorphisms. By embedding the final result in a tight cubic structure space (see Greechie (1974)) and passing to the lattice we obtain the desired result. Until the last paragraph we shall assume that $|G|>1$.

This paper was presented at the Lattice Theory Conference, Ulm, Germany, July 1975, under the title, "Every Finite Group is a Subgroup of the Collineation Group of some Projective Plane". 
Lemma 1. Let $G$ be a finite group of order at least 2 and let $L_{G}$ be the lattice constructed in Schrag $(1971,1976)$ such that $\mathrm{Aut}_{\perp}\left(L_{G}\right) \cong G$. Then

$$
\operatorname{Aut}_{\perp}\left(L_{G}\right)=\operatorname{Aut}\left(L_{G}\right) \text {. }
$$

PrOOF. We begin by recalling the construction of $L_{G}$. Schrag begins with the finite regular graph $H_{0}$ of degree 5, given by Sabidussi (1957), such that Aut $\left(H_{0}\right) \cong G$. He then takes the subdivision graph $H_{1}$ of $H_{0}$ obtained by splitting each edge in $H_{0}$ in two and for each pair of new edges adding one new point which is the common vertex of the two new edges which replace the old one (for example, a triangle becomes a hexagon). (The vertices of $H_{1}$ have degree 5 or degree 2.) Schrag then considers the graph $\mathrm{H}_{2}$ given in Fig. 1. He manufactures a family of disjoint copies of $H_{2}$-one for each element of $H_{1}$ of degree 2. Let $H_{y}$ be the copy $H_{2}$ corresponding to $y$ in $H_{1}$ of degree 2. Now he identifies the unique element (called $x$ in Fig. 1) of each $H_{y}$ of degree 2 with the corresponding $y$ in $H_{1}$ forming a

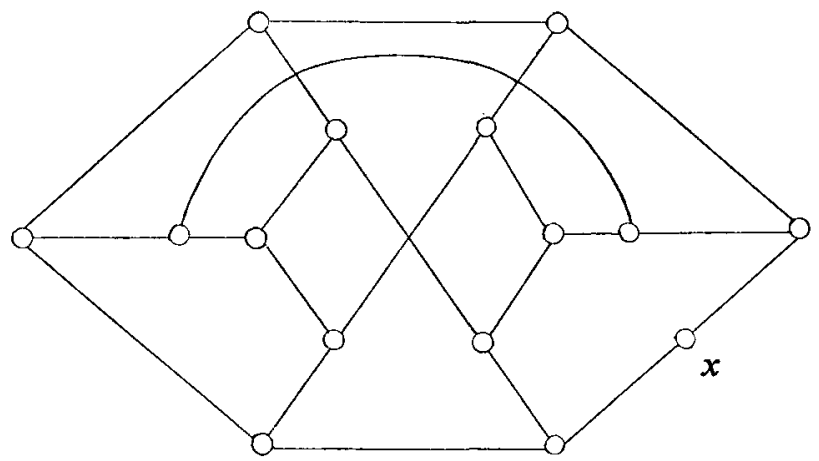

FIG. 1. Schrag's graph $\mathrm{H}_{2}$ having a one-element automorphism group.

larger graph having elements of degrees 3, 4 and 5. Call this new graph $H_{3}$. Then Aut $\left(H_{3}\right) \cong G$. The dual incidence structure $H_{3}^{*}$ has the same automorphism group. So does its logic $\mathscr{L}\left(H_{3}^{*}\right)$ which is Schrag's $L_{G}$.

Figure 2 represents the graph $\mathrm{H}_{2}$ of Fig. 1. The point $x$ has degree 2; all other points have degree 3 and are represented by triangles.

In Fig. 3 we depict the graph $H_{3}$. The circles represent the elements of degree 5 from $H_{0}$. The squares represent the elements of degree 4 obtained by identifying the element $x$ of degree 2 of $H_{y}$ with the element $y$ of degree 2 of $H_{1}$. The triangles represent the elements of $H_{2}-\{x\}$ of degree 3.

The edges of $H_{3}$ are the points of $H_{3}^{*}$ and the atoms of $\mathscr{L}\left(H_{3}^{*}\right)$. The five edges on a point of degree 5 in $H_{3}$ determine a copy of $2^{5}$ in $\mathscr{L}\left(H_{3}^{*}\right)$. These are the only sublattices of $\mathscr{L}\left(\mathrm{H}_{3}^{*}\right)$ isomorphic to $2^{5}$. Hence, a lattice automorphism $\varphi$ of $\mathscr{L}\left(H_{3}^{*}\right)$ permutes these copies of $2^{5}$ among themselves. Similarly, such $\varphi^{\prime}$ s permute the copies of $2^{4}$ in $\mathscr{L}\left(H_{3}^{*}\right)$, corresponding to the vertices of degree 4 in $H_{3}$, among themselves. However, as we shall see, there are copies of $2^{3}$ in $\mathscr{L}\left(H_{3}^{*}\right)$ which do 


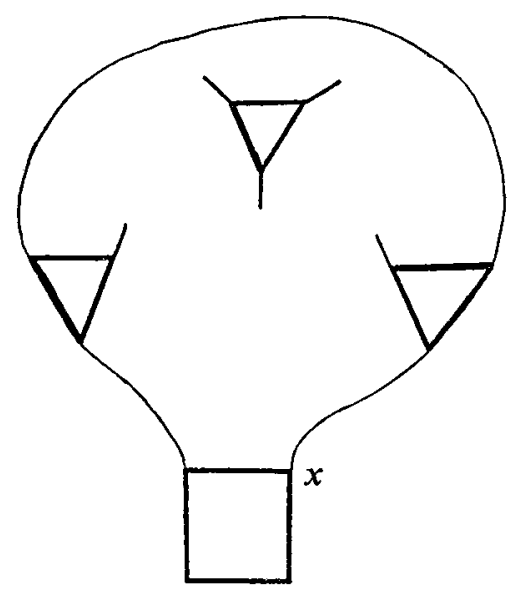

Fig. 2. An abstract version of the graph $\mathrm{H}_{2}$.

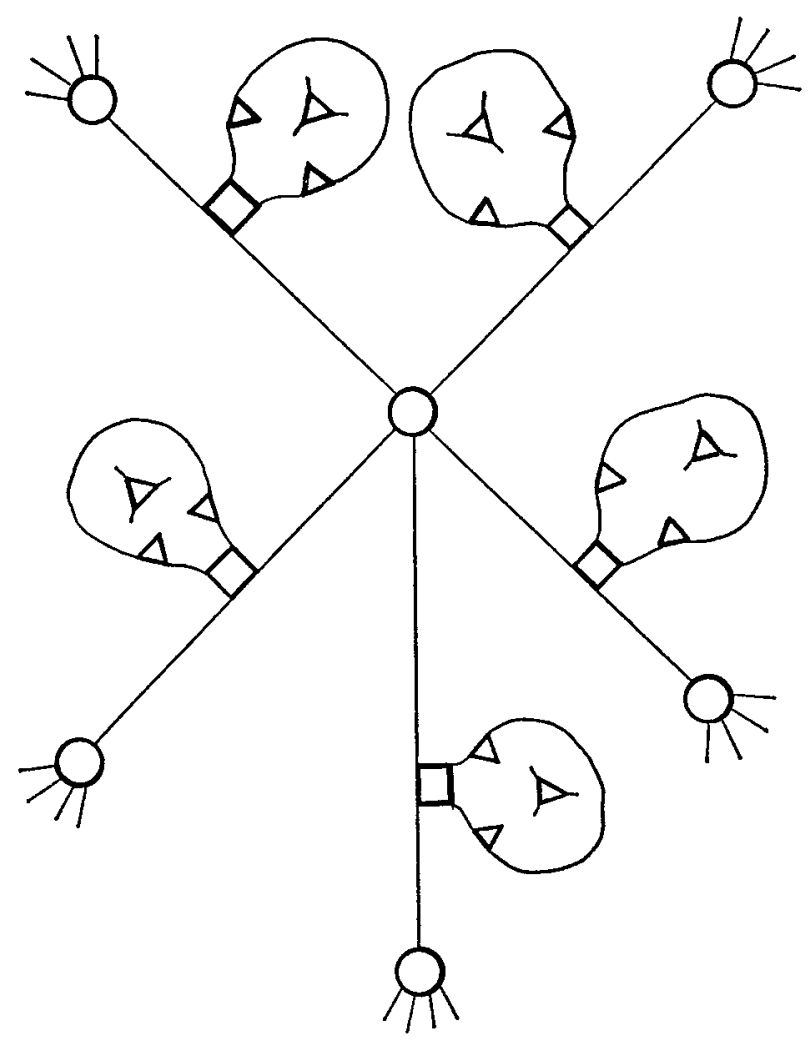

FIG. 3. A local view of the graph $H_{3}$. 
not correspond to sublattices obtained from the dual of points of degree 3 . We must therefore prove separately that there are no non-trivial lattice automorphisms of each of the sublattices determined by a single $H_{y}$ which fix the two edges adjacent to $y$ in $H_{3}$. The orthogonality space diagram for this lattice $L_{0}$ is given in Fig. 4 .

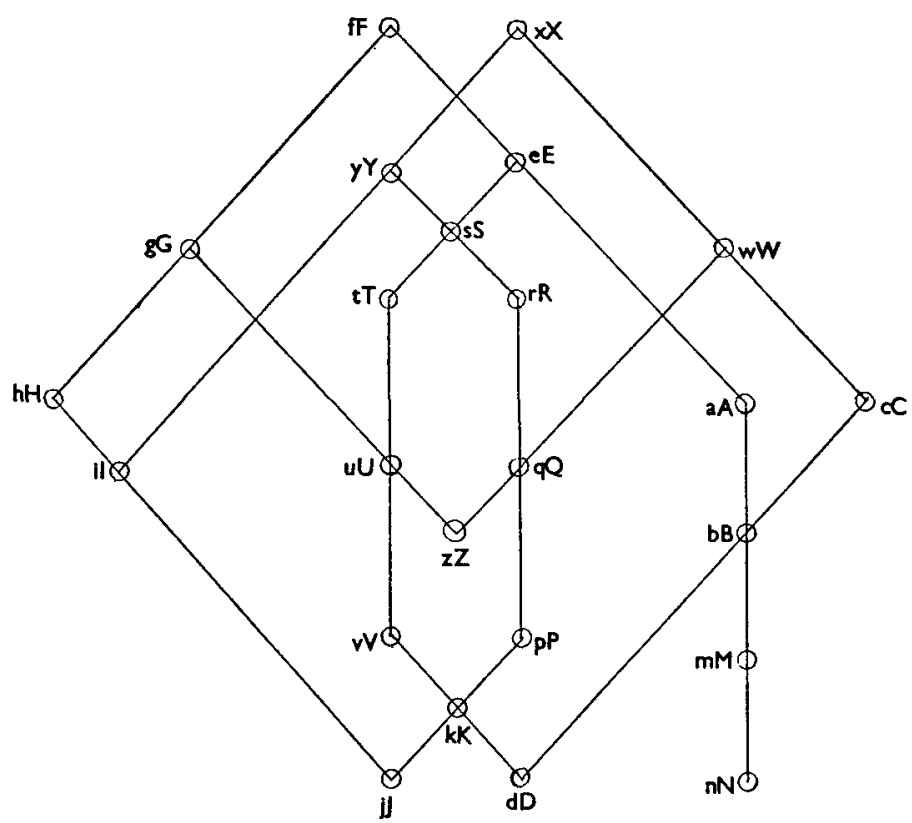

FIG. 4. The orthogonality space determined by the atoms of $L_{0}$.

$L_{0}$ has one sublattice $\beta$ isomorphic to $2^{4}$; all sublattices of $L_{0}$ of height 4 are subsets of the lattice $\beta$. We must show that $L_{0}$ contains no lattice automorphisms which fix $n$ and $m$. Since the lattice automorphisms need not preserve the orthocomplementation a priori, we must use the full force of the diagram. The reader should visualize two copies of the diagram one on top of the other. Think of the small letters as representing the elements of the bottom copy $X_{0}$; these are the atoms of $L_{0}$. Think of the capital letters as representing elements of the top copy $X_{1}$; these are the coatoms of $L_{0}$. (To complete the picture the reader should mentally visualize the six 2-element subsets of the set $\{a, b, m, n\}$ but this will not be necessary for our argument.) $L_{0}$ is an orthomodular lattice with $A=a^{\prime}, B=b^{\prime}$, etc. However, we shall ignore this fact and treat it simply as a lattice. Note that $r, S, t, U, z, Q$, together with 0 and 1 is lattice isomorphic to $2^{3}$ but not a "block" of $L_{0}$. Herein lies the problem. There may exist lattice isomorphisms which are not ortho-lattice isomorphisms. The following remark states that this is not the case.

REMARK. The only lattice automorphism of $L_{0}$ which fixes $m$ and $n$ is the identity map. 
ProOF. Let $\varphi: L_{0} \rightarrow L_{0}$ (and hence $\varphi^{-1}$ ) be a bijection preserving $\vee$ and $\wedge$ with $\varphi(m)=m$ and $\varphi(n)=n$.

We first note that (i) $t \vee d=V<1$ and (ii) $t \vee g=U<1$. By considering the unique copy of $2^{4}$ in $L_{0}$ we may infer that $\varphi(a) \in\{a, b\}$. If $\varphi(a)=b$ then $\{\varphi(c), \varphi(d)\}=\{e, f\}$ and $\{\varphi(e), \varphi(f)\}=\{c, d\}$ by considering what happens to the elements under $A$ and $B$, respectively. Since $t<E, \varphi(t) \in\{x, w, k, v\}$; but (i) above is violated unless $\varphi(d)=e$ and $\varphi(t)=v$; it follows that $\varphi(E)=D$ so that $\varphi(F)=C$ and hence $\varphi(f)=c$ and $\varphi(e)=d$. Since $h, g<F,\{\varphi(h), \varphi(g)\}=\{w, x\}$; both possible choices of $\varphi(g)$ violate (ii), since $\varphi(t)=v$. Therefore $\varphi(a) \neq b$.

It follows that $\varphi(a)=a, \varphi(b)=b, \varphi(A)=A$ and $\varphi(B)=B$. By (i) and the fact that $t \leqslant E$ we conclude that $\varphi(E) \neq F$ so that $\varphi(E)=E$; it follows that $\varphi(F)=F$ and, taking meets, $\varphi(e)=e$ and $\varphi(f)=f$. Therefore $\varphi(t) \in\{s, t\}$; by (i) again the only consistent choice of $\varphi(t)$ and $\varphi(d)$ are $\varphi(t)=t$ and $\varphi(d)=d$; hence $\varphi(C)=C$, $\varphi(D)=D, \varphi(c)=c, \varphi(s)=s, \varphi(T)=T, \varphi(S)=S$. It now readily follows that $\varphi$ is the identity function. The remark is proved.

We return to the proof of Lemma 1. Let $\varphi: L_{G} \rightarrow L_{G}$ be a lattice automorphism. Let $x$ be a vertex of $H_{0} \subset H_{3}$ so that $x$ corresponds to a copy of $2^{5}=B_{x}$ in $L_{a}=\mathscr{L}\left(H_{3}^{*}\right)$; the elements of $B_{x}$ of height 2,3 and 4 are in exactly one copy of $2^{5}$ in $L_{G}$; hence $\varphi \mid B_{x}=B_{y}$ for some vertex $y$ of $H_{0}$. Similarly, each vertex $u \in H_{1} \backslash H_{0}$ corresponds to a copy of $2^{4}=B_{u}$ in $L_{Q}$ and $\varphi \mid B_{u}=B_{v}$ for some vertex $v$ in $H_{1} \backslash H_{0}$. For each vertex $w \in H_{3} \backslash\left(H_{1} \cup H_{2}\right), w \in H_{y}$ for some vertex $y \in H_{2} \backslash H_{1}$; and $\varphi$ maps the two atoms of $B_{y}$ which are also in some $2^{5}$ to two such atoms in some $B_{z}$, $z \in H_{2} \backslash H_{1}$. Let $L_{0}^{y}$ and $L_{0}^{z}$ be the copy of $L_{0}$ corresponding to $H_{y}$ and $H_{z}$. Then $\varphi \mid L_{0}^{y}: L_{0}^{y} \rightarrow L_{0}^{y}$ is an ortho-automorphism, by the remark, and therefore $\varphi$ induces a graph isomorphism of $H_{y}$ onto $H_{z}$. It follows that $\varphi$ induces a graph automorphism $\varphi^{*}$ of $H_{3} . \varphi^{*}$ induces an ortho-automorphism $\varphi^{* *}$ on $\mathscr{L}\left(H_{3}^{*}\right)=L_{G}$ and clearly $\varphi=\phi^{* *}$. Thus $\varphi$ is an ortho-automorphism and so we have $\operatorname{Aut}\left(L_{G}\right) \subseteq \operatorname{Aut}_{\perp}\left(L_{G}\right)$. Since containment certainly goes in the other direction, we have equality.

We shall now prove the theorem. For $i=1,2$, let

$$
X_{i}=\left\{x \in L_{G} \mid \text { there exists a covering chain from } 0 \text { to } x \text { of length } i\right\}
$$

so that $X_{1}$ is the set of atoms of $L_{G}$ and $X_{2}$ is the set of elements which cover atoms. Let $X_{0}=X_{1} \cup X_{2}$ and let $\mathscr{E}_{0}=\left\{\{x, y\} \mid x \in X_{1}, y \in X_{2}\right.$ and $x \leqslant y$ in $\left.L_{G}\right\}$. Let $\left(X_{3}, \mathscr{E}_{3}\right)=\delta\left(X_{0}, \mathscr{E}_{0}\right)$ be the Dacification of $\left(X_{0}, \mathscr{E}_{0}\right)$, so that

$$
X_{3}=X_{0} \cup \mathscr{E}_{0} \text { and } \mathscr{E}_{3}=\left\{\{x, y,\{x, y\}\} \mid\{x, y\} \in \mathscr{E}_{0}\right\} .
$$

Since $L_{G}$ is a lattice, $\left(X_{3}, \mathscr{E}_{3}\right)$ is a wide cubic structure space (see Greechie (1974)) (in other words, a point closed complete Dacey space in which all maximal orthogonal sets have three elements).

Henceforth we shall utilize the definitions and notation of Greechie (1974). Since $\left|X_{2}\right|>\left|X_{1}\right|, \operatorname{Aut}\left(X_{3}, \mathscr{E}_{3}\right) \cong G$. 
Let $(Y, \mathscr{F})$ be the wide cubic structure space obtained by deleting the point $m$ (and $M)$ from Fig. 4. Note that $(Y, \mathscr{F})$ has a trivial automorphism group. Take finitely many disjoint copies of this space, one (call it $Y_{\{x, y\}}$ ) for each $\{x, y\} \in X_{3} \backslash X_{0}$, and identify the unique point of $Y_{\{x, y\}}$ which is in only one block with $\{x, y\}$ to obtain a finite wide cubic structure space $(X, \mathscr{E})$. Observe that (1) $x \in X$ implies $x$ is in at least two blocks of $\mathscr{E}$ and (2) Aut $(X, \mathscr{E}) \cong G$. Apply Theorem 2 of Greechie (1974) to embed $(X, \mathscr{E})$ in an on-trivial tight cubic structure space $\pi(X, \mathscr{E})$ so that $\mathscr{C}(\pi(X, \mathscr{E}))$ is an orthocomplemented projective plane. From (1), (2) and Proposition 3 of Greechie (1974) it follows that

$$
\operatorname{Aut}_{\perp}(\mathscr{C}(\pi(X, \mathscr{E}))) \cong G .
$$

The orthocomplementation of $\mathscr{C}(\pi(X, \mathscr{E}))$ is the absolute-point free polarity of $P=\mathscr{C}(\pi(X, \mathscr{E}))$ mentioned in the theorem. Moreover, $\operatorname{Aut}_{\perp}(\mathscr{C}(\pi(X, \mathscr{E})))$ is precisely the group of collineations of $P$ which "commute" with this polarity. $P$ is a nonDesarguesian projective plane by Corollary 2 of Greechie (1974).

The case in which $|G|=1$ is easily handled by ignoring Schrag's $L_{G}$ (which is $2^{1}$ in this case) and constructing directly a wide cubic structure space $\left(Y_{0}, \mathscr{F}_{0}\right)$ with a trivial automorphism group in which each element is in at least two blocks and then passing to $\pi\left(Y_{0}, \mathscr{F}_{0}\right)$ and $\mathscr{C}\left(\pi\left(Y_{0}, \mathscr{F}_{0}\right)\right)$. Such a space $\left(Y_{0}, \mathscr{F}_{0}\right)$ is as easy to construct as our $(Y, \mathscr{F})$ above. The theorem is proved.

The corresponding result for infinite groups may yield to the techniques of E. Mendelsohn. See, for example, "Every group is the collineation group of some projective plane", J. Geom., (1972) 2 (2), 97-106 for a similar result not involving orthogonality.

\section{References}

R. Frucht (1949), "Graphs of degree three with a given abstract group", Canad. J. Math. 1, 365-378.

R. J. Greechie (1974), "Some results from the combinatorial approach to quantum logic", Synthese 29, 113-127.

G. C. Schrag (1971), "Combinatorics and graph techniques in orthomodular theory", Ph.D. Dissertation, Kansas State University.

G. C. Schrag (1976), "Every finite group is the automorphism group of some finite orthomodular lattice", PAMS, 55 (1), 243-249.

G. Sabidussi (1957), "Graphs with given group and given graph-theoretical properties", Canad. J. Math. 9, 515-517.

\section{Department of Mathematics \\ Kansas State University \\ Manhattan \\ Kansas 66506, USA}

